\title{
The Influence of the Preoperative Status on the Risk of Postoperative Complications After Cytoreductive Surgery for Advanced-stage Ovarian Cancer
}

\author{
NICOLAE BACALBASA ${ }^{1,2,3}$, IRINA BALESCU ${ }^{4,5}$, MIHAI DIMITRIU ${ }^{1,6}$, LAURA ILIESCU $^{1,7 *}$, \\ CAMELIA DIACONU ${ }^{1,8^{*}}$, SIMONA DIMA $^{3}$, MIHAELA VILCU $^{1,9}$ and IULIAN BREZEAN ${ }^{1,9}$ \\ 1 "Carol Davila" University of Medicine and Pharmacy, Bucharest, Romania; \\ ${ }^{2}$ Department of Obstetrics and Gynecology, "I. Cantacuzino” Clinical Hospital, Bucharest, Romania; \\ ${ }^{3}$ Department of Visceral Surgery, "Fundeni” Clinical Institute, Bucharest, Romania; \\ ${ }^{4}$ Department of Surgery, "Ponderas" Academic Hospital, Bucharest, Romania; \\ ${ }^{5}$ PhD Student, "Carol Davila” University of Medicine and Pharmacy, Bucharest, Romania; \\ ${ }^{6}$ Department of Obstetrics and Gynecology, "Sf. Pantelimon" Emergency Clinical Hospital, Bucharest, Romania; \\ ${ }^{7}$ Department of Internal Medicine, "Fundeni" Clinical Institute, Bucharest, Romania; \\ ${ }^{8}$ Department of Internal Medicine, Clinical Emergency Hospital of Bucharest, Bucharest, Romania; \\ ${ }^{9}$ Department of Visceral Surgery, "I. Cantacuzino” Clinical Hospital, Bucharest, Romania
}

\begin{abstract}
Background/Aim: The aim of this study was to analyze the influence of preoperative status on the risk of developing further postoperative complications after debulking surgery for advanced-stage ovarian cancer. Patients and Methods: A total of 80 consecutive patients submitted to surgery between 2014 and 2019 for advancedstage ovarian cancer were retrospectively reviewed. Results: Among the 80 cases there were seven patients who developed serious postoperative complications requiring reintervention. Among these cases it seems that association of obesity, poor nutritional status (defined as a lower than $3.5 \mathrm{~g} / \mathrm{dl}$ serum albumin level) and elderly age were significantly associated with the risk of developing further complications. Conclusion: When selecting the candidates for debulking surgery for advanced-stage ovarian cancer attention should be focused on excluding cases presenting such preoperative risk factors in order to decrease the postoperative morbidity.
\end{abstract}

This article is freely accessible online.

*These Authors contributed equally to this study.

Correspondence to: Irina Balescu, "Ponderas" Academic Hospital, Nicolae Caramfil 85a Street, Bucharest, Romania. Tel: +40 724077709, e-mail: irina.balescu@ponderas-ah.ro

Key Words: Cytoreduction, ovarian cancer, preoperative status, morbidity.
Since Meigs intuited the principles of debulking surgery and Griffiths demonstrated them, these principles were widely investigated while the efficacy and safety of the procedure was widely studied in the last decades (1-4). In the meantime, the benefits in terms of survival have become more evident in the last years, leading to the transformation of these principles in the cornerstone of the treatment of advanced stage or relapsed ovarian cancer. However, the fact that this type of surgical approach is still associated in certain cases with significant risks of postoperative complications as well as with increased rates of perioperative death, should not be omitted. Therefore, attention was payed on determining which cases could benefit most from this surgical approach and, more importantly, which are the cases that should be excluded from surgery in order to minimize the rates of perioperative morbidity and mortality (5-8).

\section{Patients and Methods}

After obtaining approval of the Ethics Committee (no 312/2019), data of patients submitted to surgery with curative intent for advanced stage ovarian cancer between 1 January 2014 and 1 January 2019 were retrospectively reviewed. Among the identified cases we excluded patients diagnosed with non-epithelial ovarian cancer due to different biological behaviour of these tumors when compared to epithelial ovarian cancer as it has been previously demonstrated $(9,10)$; in the meantime, cases with incomplete records were also excluded. The objective of the study was to analyze the distribution of postoperative morbidity and mortality and identify potential risk factors to develop further complications among preoperative parameters. Postoperative complications were classified according to the Clavien Dindo (11) scale while the stage 
of disease was classified according to the International Federation of Gynaecology and Obstetrics (FIGO) stages (12).

\section{Results}

Between January 2014 and January 2019 we retrospectively identified 80 patients submitted to surgery with curative intent. The mean age at the time of the study was 52.6 years (range $=24-83$ years), 73 cases being diagnosed with FIGO stage IIIC disease, while the remaining seven cases were diagnosed in FIGO stage IV of disease. The median value of BMI was $28.5 \mathrm{~kg} / \mathrm{m}^{2}$ (range $=19-44 \mathrm{~kg} / \mathrm{m}^{2}$ ) while the most commonly associated comorbidities were represented by arterial hypertension (in 23 cases), diabetes mellitus (in 14 cases), chronic pulmonary obstructive disease (in five cases) and renal dysfunction (in other five cases). Preoperatively the serum albumin level was tested and proven to be lower than $3.5 \mathrm{~g} / \mathrm{dl}$ in 16 cases, while the remnant 64 cases presented higher than $3.5 \mathrm{~g} / \mathrm{dl}$ levels of serum albumin. Preoperative details of patients submitted to surgery are shown in Table I.

Among the 80 cases, ascites was present in 64 cases, with a mean volume of $1,400 \mathrm{ml}$ (range $=100-5,000 \mathrm{ml}$ ); as for the histopathological subtype, majority of cases (75) were diagnosed with serous carcinomas, followed by mucinous carcinomas (in three cases) and endometroid carcinomas (in two cases).

In all cases debulking surgery to no residual disease was tempted; however, this desiderate was achieved in 68 of the 80 cases $(85 \%)$; most commonly encountered locations which impeded obtaining complete cytoreduction were represented by diffuse mesenteric involvement (in nine cases) followed by unresectable adenopathies involving vital vascular structures (hepatic pedicle, iliac arteries - in three cases). Intraoperative details are shown in Table II.

Among the 80 patients there were seven patients who developed serious postoperative complications requiring reoperation within the first 30 days postoperatively; the reasons for re-operation were represented by postoperative intra-abdominal haemorrhage in three cases, pancreatic abscess due to pancreatic leak after distal splenopancreatectomy in one case, urinary leak after ureteral reimplantation in one case and digestive leaks followed by colorectal and respectively partial gastric resections each in one case. However, the patient who was re-operated for pancreatic leak as well as the one who developed a gastric leak died during the first month postoperatively.

Patients developing postoperative complications were significantly elderly when compared to those with uneventful evolution (67 years $v s .54$ years, $p=0.004$ ) and presented at least two associated comorbidities. In the meantime patients who developed postoperative complications necessitating reoperation had been submitted to at least two associated resections to the standard surgical approach (consisting of total hysterectomy with bilateral adnexectomy, omentectomy, lymph node dissection and peritonectomy). In the meantime association of lower or upper abdominal resections did not influence the rates of severe complications requiring reoperations). However, performing an R0 resection also seemed to influence the risk of serious postoperative complications; in fact six of seven cases developing postoperative complications had been submitted to debulking surgery to no residual disease.

As for the influence of the BMI values on the risk of postoperative complications, cases necessitating reoperation had a median BMI value of $38 \mathrm{~kg} / \mathrm{m}^{2}$, significantly higher when compared to cases with uneventful evolution (and who reported a median BMI value of $25.3 \mathrm{~kg} / \mathrm{m} 2)$. In the meantime patients who developed postoperative complications had significantly lower values of serum proteins (six of the seven patients requiring reoperation having a lower than $3.5 \mathrm{~g} / \mathrm{d}$ l level of serum albumin); among patients who did not require reoperation only 10 out of the 73 cases reported lower than $3.5 \mathrm{~g} / \mathrm{dl}$ levels of serum protein $(p=0.003)$. As for the length of hospital in stay, patients requiring re-operation reported a significantly longer hospital in stay when compared to cases in which no re-operation was needed (25 days versus 8 days, $p=0.02$ ).

We could not find any statistical correlation between the administration of neoadjuvant chemotherapy and the rates of severe postoperative complications, the rates of postoperative complications requiring reoperation being of $14.2 \%$ among patients submitted to neoadjuvant therapy and $8.2 \%$ among cases submitted to primary debulking surgery $(p=0.08)$.

\section{Discussion}

Most women with ovarian cancer are diagnosed in advanced stages of the disease, with low rates of long-term survival in the absence of a radical therapeutic strategy. However, association between complete cytoreduction and adjuvant chemotherapy significantly improved the outcomes of these patients, cases presenting a complete therapeutic response being associated with increased survival $(13,14)$. In order to maximize the debulking effort, extended resections involving both the lower and the upper abdomen and, if needed, thoracic resections have been proposed with promising results (15-17). In this context, the initial theory which sustained the fact that upper abdominal involvement is seen only in patients with a more biologically aggressive malignancy has been destroyed; one of the most reluctant studies conducted on this theme was the one published in 2006 in Gynecologic Oncology by Eisenhauer (15); the authors compared the outcomes of patients necessitating extended upper abdominal resections who were submitted to complete cytoreduction to those of patients submitted to 
Table I. Baseline characteristics of the 80 patients included in the study.

\begin{tabular}{lc}
\hline Characteristics & $\mathrm{N}=80$ \\
\hline Age (years, mean) & 52.6 \\
BMI $\left(\mathrm{kg} / \mathrm{m}^{2}\right.$, mean $)$ & 28.5 \\
Neoadjuvant chemotherapy & 73 \\
No & 7 \\
Yes & \\
Stage at diagnostic & 73 \\
FIGO IIIC & 7 \\
FIGO IV & \\
Associated comorbidities & 23 \\
Arterial hypertension & 14 \\
Diabetes mellitus & 5 \\
Chronic obstructive pulmonary disease & 5 \\
Renal dysfunction & \\
Serum albumin & 64 \\
$>3.5 \mathrm{~g} / \mathrm{dl}$ & 16 \\
$<3.5$ g/dl & \\
Histopathological subtype & 75 \\
Serous & 3 \\
Mucinous & 2 \\
Endometrial & \\
Degree of differentiation & 34 \\
Well differentiated & 14 \\
Moderately differentiated & 32 \\
Poorly differentiated & \\
\hline
\end{tabular}

standard resections for pelvic extended disease and in which complete debulking was achievable and to those of patients submitted to incomplete cytoreduction. The authors demonstrated that the overall survival was similar for cases in the first two groups and significantly higher when compared to those in the third group; therefore the median overall survival was not reached after 68 months and 84 months respectively in the first and second group respectively, significantly higher when compared to the third group (and who experienced an overall survival of only 38 months), $p<0.001$. Although not statistically significant, the rates of postoperative complications were higher for patients from the first group (12\%) when compared to the second group $(7 \%)$ and the third group $(8 \%)$. In the meantime, in patients submitted to complete cytoreduction adjuvant chemotherapy was initiated after a longer period of time; however, this aspect did not influence the long term outcomes. These data come to demonstrate that although more extended surgical procedures are usually associated with higher rates of postoperative complications and with a later onset of the adjuvant treatment the overall survival is significantly better in such cases (15). In this respect, we also chose to investigate the impact of associating upper abdominal resections on the early postoperative evolution of patients submitted to debulking surgery when compared to those submitted to standard pelvic resections. Our data
Table II. Intraoperative details of patients submitted to debulking surgery.

\begin{tabular}{lc}
\hline Characteristics & $\mathrm{N}=80$ \\
\hline Completeness of cytoreduction & \\
No residual disease & 68 \\
$<1 \mathrm{~cm}$ residual disease & 3 \\
$>1 \mathrm{~cm}$ residual disease & 9 \\
Associated visceral resections & \\
Colorectal resections & 9 \\
Urinary tract resections & 7 \\
Splenectomy & 6 \\
Atypical hepatectomy & 3 \\
Pancreatic tail resection & 3 \\
Diaphragmatic resections & 4 \\
Gastric resections & 2 \\
Small bowel resections & 16 \\
Cholecystectomy & 8 \\
Appendectomy & 7 \\
Length of surgery (minutes, mean) & 230 \\
Estimated blood loss (ml, mean) & 450 \\
Length of hospital in stay (days, mean) & 14 \\
\hline
\end{tabular}

demonstrate that the rates of perioperative complications were similar between the two groups, demonstrating that association of extended upper abdominal procedures does not induce a significant increase of the perioperative morbidity. Moreover, obtaining complete cytoreduction in such cases might associate a significant improvement of the long-term survival, which might become comparable to cases with pelvic limited disease and who are submitted to standard pelvic resections.

Therefore, attention was focused on determining which other factors might influence the risks of postoperative complications and how can they be controlled in order to minimize the perioperative morbidity. An interesting study conducted on this issue came to demonstrate that although extended pelvic and upper abdominal resections were associated with a double rate of complications when compared to standard pelvic resections this type of surgery significantly increased the disease free and overall survival (18). The study included 5,223 women submitted to debulking surgery for advanced stage ovarian cancer, with a median age of 62 years (range $=52-73$ years). Among these cases, the rate of early postoperative morbidity (within the first 30 days) was of $26.1 \%$, while the rate of postoperative mortality was of $3 \%$ and was significantly associated with age, black race, type of insurance, comorbidity burden, unscheduled admission, advanced stage (FIGO stage IV) of disease) and extensive surgical procedures. Among the comorbidities that impacted the rate of postoperative morbidity, the strongest influence was observed for chronic obstructive pulmonary disease, chronic heart failure, renal function impairment as well as obesity; however, the onset of the 
adjuvant chemotherapeutic protocol was not influenced by the apparition of postoperative complications (18). Another similar study conducted on this theme in the United States of America and published this year came to demonstrate that association of a higher rate of preoperative comorbidities, presence of ascites, increased age, higher number of platelets, necessity of administration of preoperative or postoperative transfusions as well as a higher length of surgery and disseminated lesions are associated with a more difficult postoperative evolution and an increased length of hospital in stay; in the meantime the authors underlined the fact that a longer period of hospital in stay can also increase patients morbidity as well as the overall costs (19).

As for the influence of the body mass index (BMI) on the early postoperative outcomes, it seems that this parameter is associated with significantly higher rates of postoperative complications; this effect can be explained by a direct influence of a higher BMI on the early postoperative outcomes but also by the well-known association between obesity and other comorbidities, such as cardiac or pulmonary dysfunctions or diabetes (20). In the meantime, it seems that not only the absolute value of the BMI should be taken in consideration when discussing its' influence on the early postoperative outcome. Therefore, it seems that patients presented a rapid weight loss before surgery due to the neoplastic consumption process are also at risk on developing more serious complications after extended surgical procedures for gynaecological cancer (21).

In order to provide a more appropriate selection of patients who will undergo cytoreductive surgery different prediction model and scores have been proposed with interesting results; therefore, in the study conducted by Kumar et al. on 620 patients submitted to debulking surgery, the apparition of postoperative complications was significantly influenced by age, comorbidities, BMI value, stage and surgical complexity. In this context, the authors underlined the all these factors could be considered when evaluating a risk prediction model of severe complications after debulking surgery for advanced stage ovarian cancer (22). In another similar study which investigated the effect of these parameters on the postoperative complications the authors underlined the fact the ability to predict the risk of postoperative complications increased by $27.4 \%$ when the type of procedure was analysed, by $22 \%$ when the preoperative level of albumin was analysed, by $11 \%$ when age was analysed and by $4 \%$ when associated comorbidities were analysed (19).

When studying the perioperative morbidity after debulking surgery in advanced-stage ovarian cancer another aspect which should be taken in consideration is the one regarding the type of hospital in which the patient is submitted to surgery as well as the expertise of the surgeon who performs these surgical procedures; therefore it seems that patients submitted to debulking surgery in high-volume centres and by surgeons with a good expertise in the field of gynaecological oncology, trend to have a lower rate of postoperative complications when compared to those submitted to surgery in low volume centres or in which surgery is performed by a gynaecologist with no special expertise in the field of gynaecologic oncology (23-26).

As for the association of neoadjuvant chemotherapy and interval debulking surgery, there is still an on-going debate; however, it seems that administration of neoadjuvant chemotherapy decreases the necessity of performing extended visceral resections and secondarily, decreases the rates of postoperative morbidity. However, it should not be omitted the fact that administration of neoadjuvant chemotherapy might predispose to the apparition of other types of complications such as the infectious ones due to the association of a higher degree of immunity system depression (27).

Another interesting aspect is the one related to the longterm outcomes of these patients; the question which should be raised is whether patients submitted to highly morbid surgical procedure will finally benefit from the radicality of their resections and will experience long term survival rates. Therefore, the American authors recently demonstrated that although patients developing postoperative complications had a lower rate of survival at one year follow up (the one year overall survival being of $77.2 \%$ for patients which reported postoperative complications, and $89 \%$ for patients with uneventful postoperative evolution) and despite the association between extended resections and postoperative complications; the overall survival of patients submitted to extended visceral resections was significantly improved at one year (18). In this respect, other factors influencing the poorer outcomes of patients developing postoperative complications should be investigated (28-31).

\section{Conclusion}

Debulking surgery in advanced-stage ovarian cancer is still associated with an important rate of postoperative complications; however, complete cytoreduction remains the only chance in order to provide better long-term outcomes for these patients while the perioperative outcomes seem to be also influenced by other factors such as the preoperative status. Therefore, attention should be focused on improving the preoperative status and, in extreme cases, on excluding patients with significant comorbidities in order to decrease the perioperative morbidity without affecting the completeness of the surgical gesture. In the meantime, it seems that utilisation of prediction models for postoperative complications based on the preoperative risk factors represents one of the strongest instruments in order to correctly identify preoperatively the patients at risk for developing further postoperative complications and personalize their therapeutic strategy. 


\section{Conflicts of Interest}

The Authors have no conflicts of interest to declare regarding this study.

\section{Authors' Contributions}

$\mathrm{NB}, \mathrm{MV}$, IB performed the surgical procedure; IB reviewed literature data; IB, LI, CD prepared the draft of the manuscript; IB was advisor of the surgical oncology procedures; IB reviewed the final version of the manuscript. All Authors read and approved the final version of the manuscript.

\section{Acknowledgements}

This work was supported by the project entitled "Multidisciplinary Consortium for Supporting the Research Skills in Diagnosing, Treating and Identifying Predictive Factors of Malignant Gynecologic Disorders", project number PN-III-P1-1.2PCCDI2017-0833.

\section{References}

1 Meigs JV: Tumors of the female pelvic organs. New York: Macmillen; 1934

2 Griffiths CT: Surgical resection of tumor bulk in the primary treatment of ovarian carcinoma. Natl Cancer Inst Monogr 42: 101-104, 1975. PMID: 1234624.

3 Hacker NF, Berek JS, Lagasse LD, Nieberg RK and Elashoff RM: Primary cytoreductive surgery for epithelial ovarian cancer. Obstet Gynecol 61(4): 413-420, 1983. PMID: 6828269.

4 Bristow RE, Tomacruz RS, Armstrong DK, Trimble EL and Montz FJ: Survival effect of maximal cytoreductive surgery for advanced ovarian carcinoma during the platinum era: a metaanalysis. J Clin Oncol 20(5): 1248-1259, 2002. PMID: 11870167. DOI: $10.1200 / J C O .2002 .20 .5 .1248$

5 Xu Z, Becerra AZ, Fleming FJ, Boscoe FP, Schymura MJ, Sinno AK, Morrow G, Minasian L and Temkin SM: Postoperative complications and survivorship trends following ovarian cancer surgery in New York State. Gynecol Oncol 145(1): 168, 2017 DOI: 10.1016/j.ygyno.2017.03.384

6 Bacalbasa N, Balescu I, Dima S, Herlea V, David L, Brasoveanu $\mathrm{V}$ and Popescu I: Initial incomplete surgery modifies prognosis in advanced ovarian cancer regardless of subsequent management. Anticancer Res 35(4): 2315-2320, 2015. PMID: 25862895.

7 Bacalbasa N, Balescu I, Dima S, Brasoveanu V and Popescu I: Splenectomy as part of cytoreductive surgery in recurrent epithelial ovarian cancer. Anticancer Res 35(9): 5097-5101, 2015. PMID: 26254413.

8 Bacalbasa N, Balescu I, Vilcu M, Brasoveanu V, Tomescu D, Dima S, Suciu I, Suciu N, Bodog A and Brezean I: Distal pancreatectomy en bloc with splenectomy as part of tertiary cytoreduction for relapsed ovarian cancer Proceedings of the 4th congress of the Romanian society for minimal invasive surgery in gynecology/annual days of the national institute for mother and child health Alessandrescu-Rusescu, ISI Proceedings Volume, 29-32, 2019.

9 Bacalbasa N, Taras C, Orban C, Iliescu L, Hurjui I, Hurjui M, Niculescu N, Cristea $M$ and Balescu I: Atypical right hepatectomy for liver metastasis from ovarian leiomyosarcoma a case report and literature review. Anticancer Res 36(4): 18351840, 2016. PMID: 27069167.

10 Bacalbasa N, Balescu I, Dima S and Popescu I: Ovarian sarcoma carries a poorer prognosis than ovarian epithelial cancer throughout all FIGO stages: a single-center case-control matched study. Anticancer Res 34(12): 7303-7308, 2014. PMID: 2550 3164.

11 Clavien PA, Barkun J, de Oliveira ML, Vauthey JN, Dindo D, Schulick RD, de Santibanes E, Pekolj J, Slankamenac K, Bassi C, Graf R, Vonlanthen R, Padbury R, Cameron JL and Makuuchi $\mathrm{M}$ : The Clavien-Dindo classification of surgical complications: five-year experience. Ann Surg 250(2): 187-196, 2009. PMID: 19638912. DOI: $10.1097 /$ SLA.0b013e3181b13ca2

12 FIGO Ovarian Cancer Staging. Effective Jan. 1, 2014. Available at: https://www.sgo.org/wp-content/uploads/2012/09/FIGOOvarian-Cancer-Staging_1.10.14.pdf

13 Siegel RL, Miller KD and Jemal A: Cancer statistics, 2018. CA Cancer J Clin 68(1): 7-30, 2018. PMID: 29313949. DOI: $10.3322 /$ caac. 21442

14 Jayson GC, Kohn EC, Kitchener HC and Ledermann JA: Ovarian cancer. Lancet 384(9951): 1376-1388, 2014. PMID: 24767708 DOI: 10.1016/S0140-6736(13)62146-7

15 Eisenhauer EL, Abu-Rustum NR, Sonoda Y, Levine DA, Poynor EA, Aghajanian C, Jarnagin WR, DeMatteo RP, D'Angelica MI, Barakat RR and Chi DS: The addition of extensive upper abdominal surgery to achieve optimal cytoreduction improves survival in patients with stages IIIC-IV epithelial ovarian cancer. Gynecol Oncol 103(3): 1083-1090, 2006. PMID: 16890277. DOI: $10.1016 /$ j.ygyno.2006.06.028

16 Eisenkop SM, Spirtos NM, Friedman RL, Lin WC, Pisani AL and Perticucci S: Relative influences of tumor volume before surgery and the cytoreductive outcome on survival for patients with advanced ovarian cancer: a prospective study. Gynecol Oncol 90(2): 390-396, 2003. PMID: 12893206. DOI: 10.1016/ s0090-8258(03)00278-6

17 Aletti GD, Dowdy SC, Gostout BS, Jones MB, Stanhope CR, Wilson TO, Podratz KC and Cliby WA: Aggressive surgical effort and improved survival in advanced-stage ovarian cancer. Obstet Gynecol 107(1): 77-85, 2006. PMID: 16394043. DOI: 10.1097/01.AOG.0000192407.04428.bb

18 Xu Z, Becerra AZ, Justiniano CF, Aquina CT, Fleming FJ, Boscoe FP, Schymura MJ, Sinno AK, Chaoul J, Morrow GR, Minasian L and Temkin SM: Complications and survivorship trends after primary debulking surgery for ovarian cancer. J Surg Res 246: 3441, 2019. PMID: 31561176. DOI: 10.1016/j.jss.2019.08.027

19 Smith CG, Davenport DL, Gorski J, McDowell A, Burgess BT, Fredericks TI, Baldwin LA, Miller RW, DeSimone CP, Dietrich CS, III, Gallion HH, Pavlik EJ, van NJ Jr. and Ueland FR: Clinical factors associated with longer hospital stay following ovarian cancer surgery. Healthcare (Basel) 7(3), 2019. PMID: 31277282. DOI: $10.3390 /$ healthcare 7030085

20 Kumar A, Bakkum-Gamez JN, Weaver AL, McGree ME and Cliby WA: Impact of obesity on surgical and oncologic outcomes in ovarian cancer. Gynecol Oncol 135(1): 19-24, 2014. PMID: 25110330. DOI: 10.1016/j.ygyno.2014.07.103

21 Pache B, Grass F, Hubner M, Kefleyesus A, Mathevet P and Achtari C: Prevalence and consequences of preoperative weight loss in gynecologic surgery. Nutrients 11(5), 2019. PMID: 31108841. DOI: $10.3390 /$ nu11051094 
22 Kumar A, Janco JM, Mariani A, Bakkum-Gamez JN, Langstraat CL, Weaver AL, McGree ME and Cliby WA: Risk-prediction model of severe postoperative complications after primary debulking surgery for advanced ovarian cancer. Gynecol Oncol 140(1): 15-21, 2016. PMID: 26541980. DOI: 10.1016/ j.ygyno.2015.10.025

23 Vernooij F, Heintz P, Witteveen E and van der GY: The outcomes of ovarian cancer treatment are better when provided by gynecologic oncologists and in specialized hospitals: a systematic review. Gynecol Oncol 105(3): 801-812, 2007. PMID: 17433422. DOI: 10.1016/j.ygyno.2007.02.030

24 Schrag D, Earle C, Xu F, Panageas KS, Yabroff KR, Bristow RE, Trimble EL and Warren JL: Associations between hospital and surgeon procedure volumes and patient outcomes after ovarian cancer resection. J Natl Cancer Inst 98(3): 163-171, 2006. PMID: 16449676. DOI: 10.1093/jnci/djj018

25 Diaz-Montes TP, Zahurak ML, Giuntoli RL, Gardner GJ, Gordon TA, Armstrong DK and Bristow RE: Surgical care of elderly women with ovarian cancer: a population-based perspective. Gynecol Oncol 99(2): 352-357, 2005. PMID: 16055176. DOI: 10.1016/j.ygyno.2005.06.005

26 Paulsen T, Kjaerheim K, Kaern J, Tretli S and Trope C: Improved short-term survival for advanced ovarian, tubal, and peritoneal cancer patients operated at teaching hospitals. Int J Gynecol Cancer 16(Suppl 1): 11-17, 2006. PMID: 16515561. DOI: $10.1111 / \mathrm{j} .1525-1438.2006 .00319 . x$

27 Leary A, Cowan R, Chi D, Kehoe S and Nankivell M: Primary surgery or neoadjuvant chemotherapy in advanced ovarian cancer: The debate continues. Am Soc Clin Oncol Educ Book 35: 153162, 2016. PMID: 27249696. DOI: 10.1200/EDBK_160624
28 Weidle UH, Birzele F, Kollmorgen $G$ and Rueger R: Mechanisms and targets involved in dissemination of ovarian cancer. Cancer Genomics Proteomics 13(6): 407-423, 2016. PMID: 27807064. DOI: 10.21873/cgp.20004

29 Cruz IN, Coley HM, Kramer HB, Madhuri TK, Safuwan NA, Angelino AR and Yang M: Proteomics analysis of ovarian cancer cell lines and tissues reveals drug resistance-associated proteins. Cancer Genomics Proteomics 14(1): 35-51, 2017. PMID: 28031236. DOI: 10.21873/cgp.20017

30 Fiedor E, Zajda K and Gregoraszczuk EL: Leptin receptor antagonists' action on HDAC expression eliminating the negative effects of leptin in ovarian cancer. Cancer Genomics Proteomics 15(4): 329-336, 2018. PMID: 29976638. DOI: 10.21873/cgp.20091

31 Weidle UH, Birzele F, Kollmorgen G and Nopora A: Potential microRNA-related targets for therapeutic intervention with ovarian cancer metastasis. Cancer Genomics Proteomics 15(1): 1-15, 2018. PMID: 29275359. DOI: $10.21873 / \mathrm{cgp} .20061$

Received December 9, 2019

Revised December 21, 2019

Accepted January 4, 2020 